\title{
HIV infected pregnant women monitored in the Adults 3 Department of the National Institute for Infectious Diseases "Prof. Dr. Matei Balş" - risk of mother to child transmission
}

\author{
Cristina Popescu ${ }^{1,2^{*}}$, Mariana Mărdarescu', Raluca Dulama', Raluca Mihăilescu', Daniela Munteanu', \\ Ruxandra Moroti ${ }^{1,2}$, Violeta Molagic ${ }^{1}$, Adriana Hristea ${ }^{1,2}$, Mihaela Rădulescu ${ }^{1,2}$, Cătălin Tilişcan ${ }^{1,2}$, Raluca Năstase , \\ Iulia Niculescu, ${ }^{1,2}$, Alina Lobodan1', Anca-Ruxandra Negru', Irina Lăpădat', Ligia Ionescu', Victoria Arama ${ }^{1,2}$
}

From The 9th Edition of the Scientific Days of the National Institute for Infectious Diseases Prof Dr Matei Bals Bucharest, Romania. 23-25 October 2013

\section{Background}

It was estimated that at the end of 2010 more than 34 million people are infected with HIV worldwide and 50\% of them are women. After 1989 in Romania, mother-tochild transmission of HIV remains the primary way of child infection with HIV. We analyzed mother to child transmission risk factors between 2005 and 2013.

\section{Methods}

We analyzed 18 mother-infant pairs in order to establish the risk factors for HIV transmission. We performed a retrospective study of the HIV infected women monitored in the Adults 3 Department of the National Institute for Infectious Diseases "Prof. Dr. Matei Balş".

\section{Results}

Only 8 of the pregnant women discovered the HIV infection before childbirth and received antiviral prophylaxis: 6 with combivir and lopinavir/r, 1 with combivir and atazanavir/ $\mathrm{r}$ and 1 with combivir and viramune. All of these patients had caesarean delivery and formula feeding for the child. All new-born were HIV negative. 10 women were diagnosed with HIV infection after the delivery: 9 had a vaginal delivery and one caesarean delivery. None of the women took chemoprophylaxis and all patients breastfed the children. 2 children were HIV positive. Both
HIV positive children were born from mothers with high viral loads and low $\mathrm{CD} 4$ counts.

\section{Conclusion}

All pregnant women must be HIV-tested before delivery in order to reduce the risk of mother-to-child transmission of HIV.

\section{Authors' details}

1National Institute for Infectious Diseases "Prof. Dr. Matei Balş", Bucharest, Romania. ${ }^{2}$ Carol Davila University of Medicine and Pharmacy, Bucharest, Romania.

Published: 16 December 2013

\footnotetext{
* Correspondence: crispopescu3@yahoo.com

'National Institute for Infectious Diseases "Prof. Dr. Matei Balş", Bucharest, Romania

Full list of author information is available at the end of the article
} 